\title{
A Network Celebrity Identification and Evaluation Model Based on Hybrid Trust Relation
}

\author{
Weijin JIANG, Jiahui CHEN, Yuhui XU
}

\begin{abstract}
Trust-based celebrity user identification is the key to the industry's reputation for electronic word of mouth. However, trust and mistrust are independent and coexistent concepts. In this context, we need to consider the existence of the two kinds of user relations brought about by the impact. This paper analyzes the characteristics of trust and distrust in social networks, and gives formal descriptions of trust networks, untrusted networks, and mixed trust networks. Based on the indicators such as degree distribution, correlation coefficient, and matching coefficient, the structural properties of mixed trust networks are studied. Based on the PageRank algorithm, the HTMM metrics affecting users under the mixed trust network environment are proposed. Finally, the validity of HTMM is verified through a real data set containing trust and distrust. Experimental results show that the results of HTMM's celebrity user identification method still have a low level of trust.
\end{abstract}

Keywords: celebrity user; hybrid trust; identification method; social network

\section{INTRODUCTION}

With the application and popularization of Web 2.0 technology, there have been online social networks such as Facebook, Twitter, Flickr, Sina Weibo and Renren. More and more people are willing to express their views through social networks, make friends or seek help. In this environment, companies can use the online social network in the impact of the user to expand their brand awareness, improve business sales. The study shows that $78 \%$ of the social networking community is willing to accept products or services recommended by others, and the proportion of businesses dedicated to social network marketing has increased from $23 \%$ in 2009 to $31 \%$ in 2010 [1]. So, the impact of user discovery is one of the important elements of social network analysis.

Users will face greater risk and uncertainty in virtual online social networking environments, not only to make trust the focus of attention in academia and industry, but also to make mistrust a common phenomenon in a social network environment [2]. From the overall impact of user decision-making, the role of trust and distrust alike is a mechanism for users to simplify decisions and deal with risks. However, in the user's decision-making process, mistrust does play a more important role than trust, and the effect of distrust changes on decision making is more significant [3]. User trust and mistrust constitute a user trust network [4]. Therefore, the method of user identification based on trust needs not only to consider the trust relationship among users, but also to consider the relationship between users.

However, the current user-based identification method based on trust is mainly through the construction of user trust network, according to demographic information found that affect the user, and do not consider the impact of distrust on the identification of the impact of the user. Li et al. proposed a trust mechanism to evaluate the trust values between online social network users and build user trust networks, and use artificial neural networks to identify users [5]. Zhang et al. converged with user trust networks with user reviews on network recognition in online social networks affecting users [6]. $\mathrm{Xu}$ and others based on the user trust network to study the joint influence identification of the user group, affecting the user [7]. The above study only identifies the impact of users based on the trust relationship between users. In recent years, some studies have found that the relationship between trust and distrust is to reveal the contradictory community of human interaction and exchange. It is the duality of the same thing and has distinct nature and characteristics [8-10]. In the hybrid trust network, the user receives both the favor (trust relationship) from the end user and receives the disapproval vote (no trust relationship) [11]. Therefore, the study has only considered the trust relationship between users and ignore the relationship between the user's mistrust, so that a greater impact on the impact of the user has also been identified. If the enterprise based on these users of electronic word of mouth marketing, will lead to its brand awareness and sales performance is difficult to achieve the desired goal.

This paper focuses on the impact of fusion trust and distrust on user identification methods. In addition, affecting the user through its network structure affect the online social network of other users view and decision. In this paper, we analyze the characteristics of the relationship between trust and distrust of users in hybrid trust networks, and study the structural properties of hybrid trust networks based on the degree distribution of trust and distrust, the correlation coefficient of trust and distrust, and the hybrid model.

Based on the PageRank algorithm, the HTMM index of the influencing user's influence in the hybrid trust network environment is designed by combining the trust network and the distrust network. The method of recognizing the user based on HTMM is put forward. Finally, the validity of the method is verified by comparing the performance of user identification method and benchmark method based on HTMM in real social network data set.

\section{RESEARCH ON TRUST AND DISTRUST IN DIFFERENT DISCIPLINES}

Since the sixties of the twentieth century, trust as a mechanism for simplifying the relationship complex has attracted the attention of scholars of different disciplines. Researches have a deeper understanding and understanding of the definition, nature, function and type of trust, especially the origin of trust, the origin of personality and the origin of culture, and formed more consensus. Based on 
the relevant research, we introduce the research on trust in different disciplines.

Sociology believes that trust is an aspect that all social relations will contain, which indicates the expectations of the future. It is more concerned about the behavioral interaction between individuals, emphasizing the impact of social interaction on trust [12-14]. And psychology has studied the trust from the psychology and behavior [15]. From a psychological point of view, the traditional psychologists believe that trust is a belief, expectation or deeply rooted in the personality of the human emotions, is a social learning through the formation of relatively stable personality traits. From the point of view of behavior, social psychology holds that trust is the individual psychology and behavior generated by the specific context stimulus. In contrast to sociology and psychology of trust research, economics advocates economic rationality, and believes that trust is the result of individual rational calculation, which treats trust as computational trust [16]. In addition, the study of management argues that trust is an interactive bilateral relationship, and emphasizes that trust is the expectation of the believer's behavior on the behavior of the believer and has an impact on the interest of the believers [17]. In the field of information science scholars believe that if an entity is credible, then all the behavior of the entity and its results are predictable, the behavior state is measurable, the behavioral results are assessable, behavioral abnormalities are controllable. The credibility of an entity is described mainly from the perspective of identity credibility, ability trustworthiness and behavior trustworthiness [18-20].

Contrary to the positive study of trust, scholars are less concerned about the opposite of trust that is distrust. This is mainly because people have always believed that distrust is the opposite of trust, with similar causes and consequences [19]. Therefore, people think that understanding the trust can understand the mistrust. In recent years, however, researchers have found that distrust, as the opposite of trust is not a simple antithesis to the same structure of trust as is traditionally known, but rather a concept that separates and coexists with each other [20].

From the different disciplines of trust and mistrust research can be found, psychologists, according to the psychological paradigm of the traditional paradigm, understand trust/mistrust as individual psychological events, focus only on cognitive and distrustful cognitive content and behavioral performance, without considering the impact of social environmental factors [23]. And sociologists believe that trust and mistrust are the same important concepts in social relations and are social phenomena associated with social structures and cultural norms. It studies both trust and mistrust among individuals, and studies the trust and mistrust between large groups of social groups, while focusing on the role of trust and mistrust. Management scholars believe that trust/mistrust is the result of an expectation and interaction between individuals or between individuals and organizations. On the basis of the above research results, this paper also considers the relationship between trust and distrust, studies the structural nature of hybrid trust network, finds the relationship between trust and mistrust of online social network users, and puts forward the method of recognizing users in hybrid trust network environment.

\section{THE METHOD OF USER IDENTIFICATION IN HYBRID TRUST NETWORK ENVIRONMENT \\ 3.1 Statement of Problem}

According to the above research, there are both trust and distrust in the social network environment. Identify the negative impact of the larger users, the impact of the user there is more distrust of the end user, indicating that the user's point of view is difficult to be accepted by other users. Therefore, it is necessary to integrate user trust relationship and non-trust relationship, and propose a novel method to adapt the user identification method in hybrid trusted network environment. This paper presents a formal description of the concept of user trust network, user distrust network, hybrid trust network and so on. On this basis, the research question of this paper is described as follows:

Definition 1 User Trust Network $U T N=\left(V T, T, W_{t}\right)$ is a 3-tuple, and $V T$ represents the node set that the user trusts the network; $T=\left|\left(u_{i}, u_{j}, \omega_{t}\right)\right| u_{i} \in V T \wedge u_{j} \in V T \wedge \omega_{t} \in W_{t}$ denotes the trust relationship between the user trust network nodes, $u_{i} T u_{j}$ denotes the node $u_{i}$ trust node $u_{j} ; W_{t}$ denotes the trust strength among the nodes in the trusted network.

Definition 2 User Distrust Network $D I N=\left(V D, D, W_{d}\right)$ is a 3-tuple, and $V D$ represents the node set that the user distrusts the network; $D=\left\{\left(u_{i}, u_{j}, \omega_{d}\right) \mid u_{j} \in V D \wedge u_{j} \in V D \wedge\right.$ $\left.\omega_{d} \in W_{d}\right\}$ indicates the distrust between nodes that do not trust the network, $u_{i} D u_{j}$ indicates that node $u_{i}$ does not trust the node $u_{j} ; W_{d}$ indicates that there is no trustworthiness between nodes in any network.

Definition 3 Hybrid Trust Network $M T N=(V, T, D$, $\left.W_{t}, W_{d}\right)$ is a 5-tuple, where $V$ represents the set of nodes of the hybrid trust network; $\left.T=\left\{\left(u_{i}, u_{j}\right) \mid u_{i} \in V \wedge u_{j} \in V\right)\right\}$ represents the trust relationship between nodes in a hybrid trust network. $u_{j} T u_{i}$ represents the node $u_{i}$ trust node $u_{j} ; D$ $\left.=\left\{\left(u_{i}, u_{j}\right)\right\} \mid u_{i} \in V \wedge u_{j} \in V\right\}$ is the distrust relationship between the nodes in the hybrid trust network. $u_{i} D u_{j}$ represents the node $u_{i}$ distrust node $u_{j} ; W_{t}$ represents the trust strength between nodes in a mix trust network; $\mathrm{W}_{\mathrm{d}}$ represents the distrust between nodes in a hybrid trust network. Obviously, $U T N \subseteq M T N, D T N \subseteq M T N$, i.e. user trust network and user distrust network is a subnet of hybrid trust network. A hybrid-trusted network environment affects user identification. Suppose that $M T N=\left(V, T, D, W_{t}\right.$, $W_{d}$ ) represents a hybrid trust network that contains a user trust relationship and a distrust relationship. $u_{1}, u_{2}, \ldots, u_{n} \in$ $V$ denotes a user in a hybrid-trusted network environment, which requires that the Top $k$ influencing users $\left\{I_{1}, I_{2}, \ldots\right.$, $\left.I_{k}\right\}$ be identified from $\left\{u_{1}, u_{2}, \ldots, u_{n}\right\}$ according to the influence rank; satisfying $\operatorname{In} f_{1}>\operatorname{In} f_{2}>\ldots>\operatorname{Inf} f_{k}$, where $T$ is hybrid trust network user influence, $j \in\{1,2, \ldots, k\}$.

\subsection{Structural Analysis of Hybrid Trust Networks}

In order to study the recognition method of users in hybrid trust network environment, firstly, the characteristics of trust relationship and distrust relationship are analyzed, and the structural properties of hybrid trust network are studied. In this paper, four kinds of indicators are used to study the structural properties of hybrid trust networks, including the degree distribution of hybrid trust networks, the correlation coefficients between trust and distrust under hybrid trust networks, the cumulative distribution of distrust 
and trust ratios under hybrid trust networks and the coefficients of trust and distrust under hybrid trust networks. The four indicators are described in detail below.

\subsubsection{Degree Distribution of Hybrid Trust Networks}

The degree distribution of hybrid trust networks includes trust distribution and distrust distribution. Its formal description is shown in Eq. (1) and (2),

trustDegree $(u)=\sum$ trustee $(u)$

Where trustee $(u)$ represents the frequency at which the credit user $u$ is trusted by other users, and the end user is the other online user directly connected to the credit user $u$.

$$
\operatorname{distrustDegree}(u)=\sum \operatorname{distrustee}(u)
$$

Where distrustee $(u)$ refers to the frequency at which the credit user $u$ is not trusted by other users.

\subsubsection{The Correlation Coefficient between Trust and Distrust}

In the hybrid trust network environment, the relationship between trust and distrust can be calculated by the linear relationship between trust and distrust, i.e., the correlation coefficient, as shown in Eq. (3).

$$
\operatorname{corr}=\frac{\sum_{i=1}^{n}\left(k_{t}^{i}-\overline{k_{t}}\right)\left(k_{d}^{i}-\overline{k_{d}}\right)}{\sqrt{\sum_{i=1}^{n}\left(k_{t}^{i}-\overline{k_{t}}\right)^{2}} \sqrt{\sum_{i=1}^{n}\left(k_{d}^{i}-\overline{k_{d}}\right)^{2}}}
$$

Where corr denotes the correlation coefficient; $k_{t}$ denotes the trust degree of the user $u ; \bar{k}$ denotes the average value of the user's trust; $k_{d}$ denotes the degree of non-trust of the user $u$; and $\bar{k}_{d}$ denotes the average value of the user's non-trust.

\subsubsection{Cumulative Distribution of Trust and Distrust Ratio}

In order to further study the correlation between trust and distrust in the hybrid trust network environment, we can calculate the cumulative distribution of trust and distrust ration. As shown in Eq. (4), where $R(p)$ represents the cumulative distribution of trust and unbelief rations, $k_{t}$ represents the trust of the user, and $k_{d}$ represents the user's unbelief.

$$
R(p)=\frac{k_{t}}{k_{d}}>P
$$

According to the Eq. (3) and (4), if the user's trust is weakly related to the distrust and most of the user's trust is higher than its distrust, it means that in a hybrid-trusted network environment, the user is more inclined to trust users with high confidence.

\subsubsection{Hybrid Mode}

The hybrid mode is used to measure the probability that a user with a trust degree $k_{r}$ is connected to a user who does not trust $k_{d}$, including the correlation function $k_{n n}$ and the co-ordinate of trust and distrust. The correlation function $k_{n n}$ of trust and distrust is measured by the mapping between the degree of trust and the average degree of distrust of all nodes with the trust, as shown in Eq. (5).

$$
\begin{aligned}
& k_{n n}(\text { kout })=\frac{1}{|u|(u, v)=k o u t} \sum u \mid k u= \\
& =k o u t \frac{1}{|v|(u, v) \in A} \sum V \mid(u, v) \in A k_{i n}^{V}
\end{aligned}
$$

Correspondingly, the co-ordinate is defined as the Pearson correlation coefficient between users, where $j_{i}$ and $k_{i}$ represent the degrees of the nodes at both ends of the $i^{\text {th }}$ arc (including trust and distrust, $i=1, \ldots, M)$. As shown in Eq. (6).

$$
r=\frac{\frac{1}{M} \sum_{i=1}^{n} j_{i} \times k_{i}-\left[\frac{1}{M} \sum_{i=1}^{n} \frac{1}{2}\left(j_{i}+k_{i}\right)\right]^{2}}{\frac{1}{M} \sum_{i=1}^{n} \frac{1}{2}\left(j_{i}^{2}+k_{i}^{2}\right)-\left[\frac{1}{M} \sum_{i=1}^{n} \frac{1}{2}\left(j_{i}+k_{i}\right)\right]^{2}}
$$

According to the Eq. (5), if the degree of trust and distrust is an upward trend, it shows that there is a central authority in the hybrid trust network environment. According to the Eq. (6), if the co-ordinate is positive, it indicates that the nodes with high degree of social networking are more likely to connect, that is to say with homology. If the co-ordinate is negative, it indicates that nodes with small values are more likely to have high connectivity values, that is, hybrid trust networks have heterogeneity.

\subsection{The Method of User Identification Based on Hybrid Trust Network \\ 3.3.1 The Index of User Influence under Hybrid Trust Network}

The metrics that affect the user are measures that measure the size of the user's influence in the social network. The existing commonly used degree of central indicators only consider the homogeneity of the relationship between users, do not consider the heterogeneous relationship, such as hybrid trust network contains both trust and distrust relationship. To this end, we need to design a new user impact metric that adapts to a hybrid trust network.

The PageRank algorithm is primarily used to measure the importance of a particular page in the Internet relative to other pages in the search engine [21]. The algorithm is also widely used to assess the importance of users in the online social network [22]. The value of a page's PageRank (i.e., the number of votes) is derived from the importance of all pages linked to it through a recursive algorithm. In this algorithm, the association between web pages is only chain in and out. However, in a hybrid-trusted network 
environment, the vast majority of users have both trust and distrust. Assume that the trust relationship indicates that the vote is in favor of the vote and does not trust to vote against it. In this case, the PageRank algorithm cannot be used directly to measure the importance (influence) of the user in a hybrid-fed network environment. Therefore, based on the PageRank algorithm, this paper proposes a hybrid trust modeling method (HTMM) indicator, which is used to measure the importance of users in a hybrid-fed network environment. The detailed calculation method is described as follows.

Suppose Lucy, Ada, Alice, Bob and David form a social network, as shown in Fig. 1.

The red arrow and the black arrow in Fig. 1 represent the relationship of trust and distrust between the users. The following describes how to calculate the HTMM value of Alice's hybrid trust as an example. Alice received trust from three users, such as Lucy, Ada and Bob, and got a distrust from David. Bob trusts Lucy and Alice. Similarly, Lucy trusts both Ada and Alice. David believes that Bob includes three other users, including Alice. Then, Alice's MTPT values are calculated as follows.

\section{$H T M M($ Alice $)=H T M M($ Ada $)+H T M M($ Lucy $) / 2+$ $+\operatorname{HTMM}(\mathrm{Bob}) / 2-H T M M($ David $) / 3$.}

By combining the above calculation process, the HTMM value of any user in the hybrid trust network environment can be calculated by the Eq. (7).

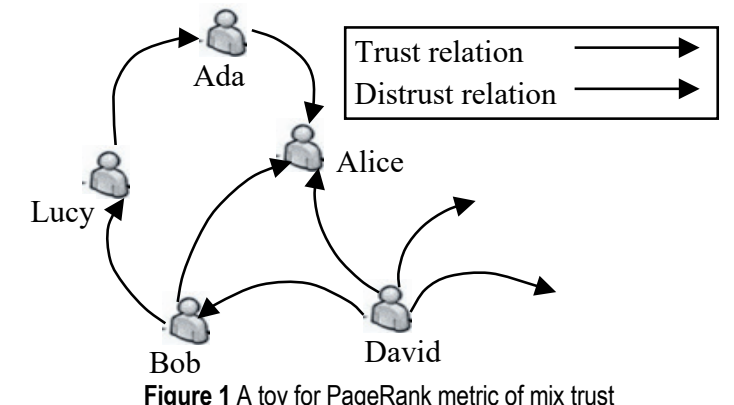

$$
H T M M=\sum_{u j \in T_{j}} \frac{\operatorname{MTPR}\left(u_{j}\right)}{\text { trustDgree }_{j}}-\sum_{u k \in D_{i}} \frac{\operatorname{MTPR}\left(u_{k}\right)}{\text { trustDgree }_{k}}
$$

Among them, HTMM $(u j)$ that the user $u_{i}$ hybrid trust PageRank value, trustDegree ${ }_{j}$ and distrustDegree $k_{k}$ represent the $u_{j}$ and $u_{k}$ 's trust and distrust respectively. $T_{j}$ represents the user set of the trusted user $u_{i}$, and $D_{i}$ represents the user set that does not trust the user $u_{j}$. Therefore, by fusing trust and distrust, the HTMM index proposed in the paper can adapt to the measurement of user influence in a hybrid trust network environment.

\subsubsection{User Identification Method Based on HTMM}

The user-based identification method based on the impact of the user is based on the user's trust in the network structure, by estimating the importance of the user to discover the impact of the user. However, the hybrid trust network environment has both trust between users and no relationship. Therefore, considering only the trust relationship and ignoring the relationship of distrust will lead to the recognition of a higher negative impact on the impact of the user. To solve this problem, this paper improved PageRank algorithm [32] in the field of search engine, according to the characteristics of user trust and distrust between the hybrid trust network, proposed a user identification method based HTMM in hybrid trust network, as shown in algorithm 1 .

Algorithm 1 User Identification Method Based on HTMM

Input: User trust list Trust-list; user does not trust list Distrust-list

Output: Hybrid trust in the network of user rankings

Variable: number of previous $K$ users

1. $\boldsymbol{T N} \times \boldsymbol{N}, \boldsymbol{D} \boldsymbol{N} \times \boldsymbol{N}$ and $\boldsymbol{G} \boldsymbol{N} \times \boldsymbol{N}$ are adjacency matrices that are used to store trusted networks, distrust networks, and hybrid trust networks, respectively, and $\mathrm{N}$ is the number of users of hybrid trust networks.

2. for $\mathrm{i}=1$ to $\mathrm{N}$ do

3. for $\mathrm{j}=1$ to $\mathrm{N}$ do

4. if (ui, uj) $\in$ Trust-list

5. tif $=1$; // Calculate the adjacency matrix of the trust network

6. else tif $=0$;

7. if (ui, uj) $\in$ Distrust-list

8. Dif $=1$; // Calculate the adjacency matrix of the distrust network

9. else tij $=0$;

10. end for

11. for $\mathrm{i}=1$ to $\mathrm{N}$ do

12. for $\mathrm{j}=1$ to $\mathrm{N}$ do

13. gij=tij-Dij; // Calculate the adjacency matrix of hybrid trust network

14. end for

15. for $\mathrm{i}=1$ to $\mathrm{N}$ do

16. for $\mathrm{j}=1$ to $\mathrm{N}$ do

17. $\mathrm{C}[\mathrm{i}]=\mathrm{c}[\mathrm{i}]+\mathrm{g}[\mathrm{i}][\mathrm{j}] ; / /$ Calculate the column sum of adjacency matrices in hybrid trust network

18. $r[i]=r[i]+g[j][i] ; / /$ Calculate the row sum of adjacency matrices in hybrid trust network

19. end for

20. for $\mathrm{i}=1$ to $\mathrm{N}$ do

21. for $\mathrm{j}=1$ to $\mathrm{N}$ do

22. $\delta \mathrm{ij}=$ gijdtv c[i]; // Calculate the state transition matrix of Markov

23. end for

24. Calculate equation $\alpha=\left(\left(\mathrm{X}_{1}, \ldots, \mathrm{X}_{\mathrm{i}}\right) \mathrm{TA} \alpha=\alpha, \sum_{i} X_{i}=1 / /\right.$ $\alpha$ denotes the steady-state probability, defined as the HTMM vector under the hybrid trust network, $X_{i}$ denotes the influence of the $i^{\text {th }}$ user.

25 . Sort the descending order of $\alpha$, output top $k$ users

The algorithm mainly expresses the hybrid trust network as a directed graph, where each user represents the node of the directed graph; the trust/distrust relationship between the users is the directed edge of the directed graph. Assuming that the user in the hybrid trusted network chooses to trust/distrust the next user's process regardless of the user who has trusted/distrusted the user in the past, it only depends on the user who is currently trusted/distrusted. Then the selection process of the user in the hybrid trust network environment can be considered as a stochastic 
process of finite state discrete time, and the state transition process can be studied by using the user identification method which affects the influence of trust and distrust in Markov Chair. Through the equation of step 24, we can compute the HTMM values of hybrid trusts for each user's steady state. The time complexity of the algorithm is $\mathrm{O}(\mathrm{N} 2)$.

\section{EXPERIMENTAL STUDY \\ 4.1 Experimental Data Set}

The experiment uses Paolo Massa to obtain a public data set for social networking Epinions.com [22]. Unlike the data collected by Richardson from the University of Washington, Paolo Massa's data collection not only contains trust relationships among users, but also contains distrust relationship between users. This paper provides a data base for the study of the structural nature of hybrid trust networks and the methods that affect user identification in a hybrid trust network. The author has studied the statistical properties of the data and validated the sample set as the sample validity of the Epinions.com and has been applied in the field of trust-based recommendation systems [21]. The data set contains three files, namely, trust/distrust information, comment author information and comment score information. The data set contains 131829 users; 841372 trust or distrust relationships, of which 717667 trusts, 123705 distrusts; 85000 users get at least one trust or distrust relationship; 1560144 reviews; 13668319 reviews score.

\subsection{Research on the Structural Properties of Hybrid Trust Network}

Recent studies have shown that trust and mistrust are revealing the relationship between human interaction and exchange of contradictions, with different nature and characteristics. According to the analysis method of hybrid trust network structure given in Section 3, we use the cumulative distribution of trust degree and distrust degree, the cumulative distribution of trust and distrust rate and the hybrid model to study the relationship between user trust and distrust in hybrid trust network.

Fig. 2 shows the trust and distrust distribution of users under hybrid trust networks. It can be seen that the user's distrust and trust are subject to the law of power distribution, consistent with the literature [18], which the user's trust degree is 5.444, the variance is 1045.772; and the unequal reliability is 0.9383 , the variance is 30.1014 . The experimental results show that the distrust relationship among users is also widespread in the user trust network, and there are different degrees of trust and distrust. The above network characteristics indicate that there is a central node user in a hybrid trust network, which can influence the views or decisions of other users through the network structure. These findings suggest that the impact of the user's presence in a hybrid trust network and the need to consider the impact of distrust relationships on the impact of users.

The correlation coefficient between user trust and mistrust calculated according to Eq. (3) is 0.3704 ( $p$-value $b \leq 0.001)$. The results show that the trust of the user under the hybrid trust network is not strongly related to its distrust. In addition, Fig. 3 shows the cumulative distribution of the trust and distrust ratio of users under hybrid trust networks. According to the experimental results, when the ratio of trust and distrust is $0.001,0.01,0.1$ and 1 , the cumulative distribution function values are $46.24 \%, 46.26 \%, 52.88 \%$ and $63.46 \%$. It can be seen that most of the user's trust is higher than its distrust, and only a small part of the user's trust is higher than the degree of distrust. The results of this study show that users with low trust in the hybrid trust environment are difficult to obtain the trust of other users, that is, most users are likely to favor users with higher trust than users who are not trustworthy. In order to further validate this observation, the hybrid model and the matching coefficient under the hybrid trust network are studied below.
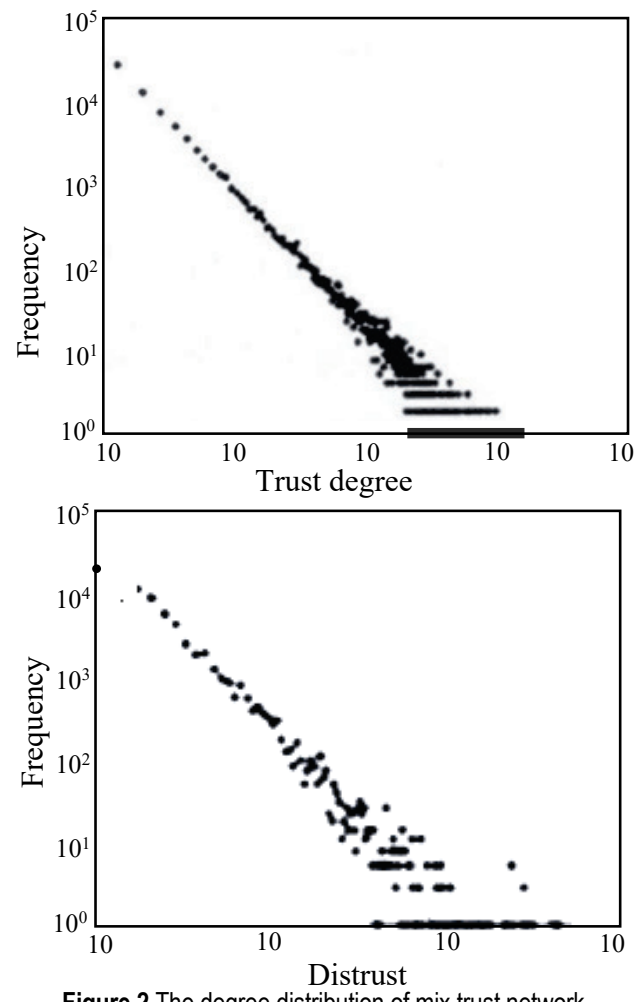

Figure 2 The degree distribution of mix trust network

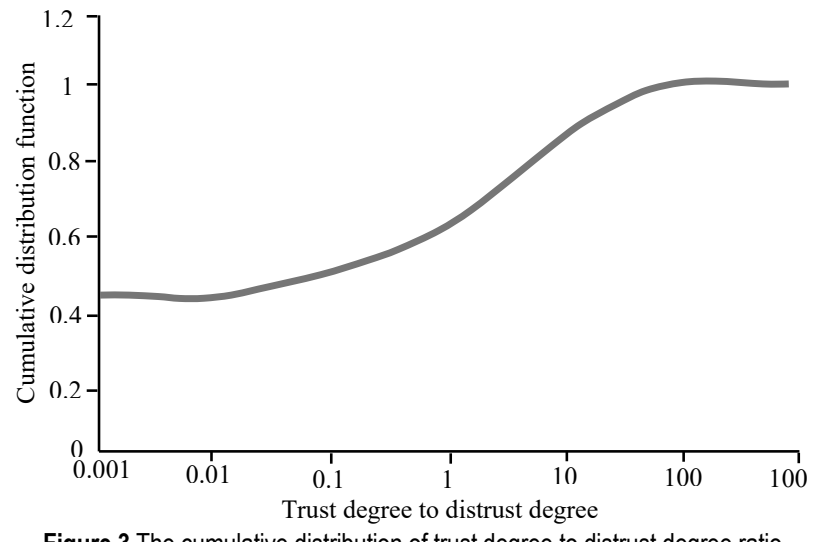

Figure 3 The cumulative distribution of trust degree to distrust degree ratio

Fig. 4 and Fig. 5 show the results of the correlation function $k_{t}$ of user trust and distrust in hybrid trust network, where Fig. 4 shows the mapping between trust and the average degree of distrust of all nodes with this trust. In the Fig. 4, the abscissa indicates the degree of trust, and the ordinate indicates the average of the degree of distrust. 


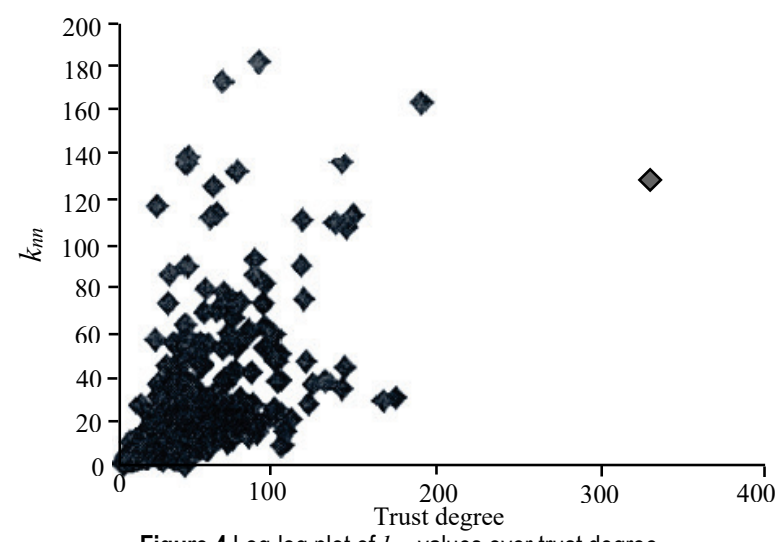

Figure 4 Log-log plot of $k_{n n}$ values over trust degree

It can be seen from Fig. 4 that the user with low trust degree is also low and the user with high trust still maintains a low degree of distrust. In addition, Fig. 5 shows the mapping between the degree of distrust and the average trust of all nodes with the distrust. Among them, the abscissa indicates the degree of distrust, and the ordinate indicates the average of the degree of trust. It can be seen from Fig. 5 that the user with high confidence is less trustworthy; when the degree of distrust increases, the average degree of trust will gradually become less. The experimental results in Fig. 4 and Fig. 5 show that users are more likely to believe in trustworthy users rather than those who do not trust. The study found that the trust relationship based on the identification of users not only need to consider the impact of the user's trust relationship, but also need to consider the impact of the user's relationship.

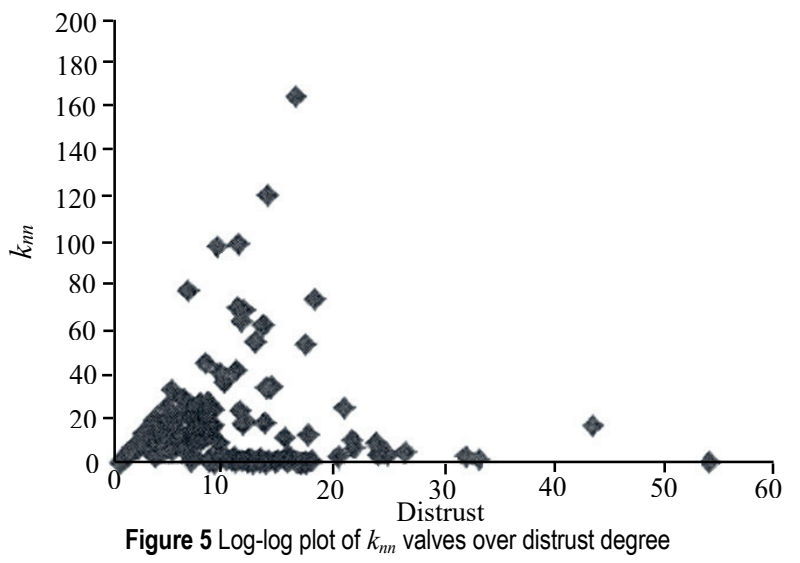

\subsection{Influential User Identification and Evaluation in Hybrid Trust Network Environment}

In order to verify the validity of the influential user identification method based on HTMM, we choose the algorithm based on PageRank algorithm, degree centered algorithm [22] and so on, and compare them with the method proposed in the paper. The centrality algorithm uses the centrality index to express the user's influence; the PageRank algorithm uses the PageRank value to measure the user's influence; and the HTMM algorithm uses the HTMM value to indicate the user's influence size. The experimental results are shown in Tab. 1 and Tab. 2, respectively [23].

Table 1 The rank of top 20 influencers in mix trust networks

\begin{tabular}{|c|c|c|c|c|c|c|}
\hline \multirow{2}{*}{ Rank } & \multicolumn{2}{|c|}{ Based on the degree of center method } & \multicolumn{2}{|c|}{ Based on the PageRank method } & \multicolumn{2}{|c|}{ Based on the HTMM method } \\
\hline & User Id & Influence & User Id & Influence & User Id & Influence \\
\hline 1 & 205639 & 1673 & 252420 & 14.67 & 295491 & 9.87 \\
\hline 2 & 200118 & 1536 & 480385 & 10.28 & 205639 & 9.74 \\
\hline 3 & 252420 & 1464 & 295491 & 9.87 & 346777 & 8.61 \\
\hline 4 & 253067 & 1295 & 205639 & 9.74 & 306614 & 8.22 \\
\hline 5 & 200338 & 1252 & 346777 & 8.61 & 405503 & 7.84 \\
\hline 6 & 295491 & 1246 & 306614 & 8.22 & 562458 & 7.71 \\
\hline 7 & 223677 & 1225 & 405503 & 7.84 & 667808 & 7.66 \\
\hline 8 & 204418 & 1222 & 562458 & 7.71 & 471240580 & 7.47 \\
\hline 9 & 346777 & 1190 & 667808 & 7.66 & 200118 & 7.19 \\
\hline 10 & 243427 & 1139 & 471240580 & 7.47 & 302444 & 7.02 \\
\hline 11 & 204441 & 1067 & 200118 & 7.19 & 394804 & 7.00 \\
\hline 12 & 209674 & 1040 & 302444 & 7.02 & 249990 & 6.94 \\
\hline 13 & 355176 & 1027 & 394804 & 7.00 & 243427 & 6.92 \\
\hline 14 & 234885 & 1010 & 249990 & 6.94 & 3239350148 & 6.84 \\
\hline 15 & 207186 & 1001 & 243427 & 6.92 & 232924 & 6.81 \\
\hline 16 & 335034 & 989 & 3239350148 & 6.84 & 257170 & 6.79 \\
\hline 17 & 200500 & 936 & 232924 & 6.81 & 372200 & 6.71 \\
\hline 18 & 262868 & 839 & 257170 & 6.79 & 335034 & 6.65 \\
\hline 19 & 480385 & 890 & 372200 & 6.72 & 223677 & 6.63 \\
\hline 20 & 372535 & 872 & 335034 & 6.65 & 253067 & 6.52 \\
\hline
\end{tabular}

Tab. 1 lists the ranking of Top 20 impact users who have been identified by the three methods of user identification in the hybrid trust network. The user ID in the Tab. 1 represents the identification number of the user that affects the user under the hybrid trust network, and the influence in the Tab. 1 indicates the size of the user's influence through the above-mentioned different indicators. The data from the Tab. 1 can be found in the Top 20 impact users, the three affect the user identification method found a different impact on the user. For example, user \#200338 is recognized as influencing a user in a degree centrality approach; however, it does not appear in the PageRank and HTMM methods. In addition, among the Top 20 impact users, the same impact users found have different rankings. For example, the impact of user \#295491 is ranked first in the HTMM method, but ranked 6th and 3rd respectively based on the degree-centric approach and based on the PageRank method; affecting user \#200118 ranked second in the center-based approach, and ranked on the PageRank method and the HTMM method are $11^{\text {th }}$ and $9^{\text {th }}$ respectively. 


\begin{tabular}{|c|c|c|c|c|c|c|c|c|c|c|c|c|c|c|}
\hline \multirow[b]{2}{*}{ Algorithm } & \multicolumn{2}{|c|}{ Top 10} & \multicolumn{2}{|c|}{ Top 20} & \multicolumn{2}{|c|}{ Top 30} & \multicolumn{2}{|c|}{ Top 40} & \multicolumn{2}{|c|}{ Top 50} & \multicolumn{2}{|c|}{ Top 80} & \multicolumn{2}{|c|}{ Top 100} \\
\hline & $\begin{array}{c}\text { Trust } \\
\text { degree }\end{array}$ & \begin{tabular}{|c|} 
Distrust \\
degree
\end{tabular} & $\begin{array}{c}\text { Trust } \\
\text { degree }\end{array}$ & $\begin{array}{c}\text { Distrust } \\
\text { degree }\end{array}$ & $\begin{array}{c}\text { Trust } \\
\text { degree }\end{array}$ & $\begin{array}{c}\text { Distrust } \\
\text { degree }\end{array}$ & $\begin{array}{c}\text { Trust } \\
\text { degree }\end{array}$ & \begin{tabular}{|c|}
$\begin{array}{c}\text { Distrust } \\
\text { degree }\end{array}$
\end{tabular} & $\begin{array}{c}\text { Trust } \\
\text { degree }\end{array}$ & $\begin{array}{c}\text { Distrust } \\
\text { degree }\end{array}$ & $\begin{array}{c}\text { Trust } \\
\text { degree }\end{array}$ & $\begin{array}{c}\text { Distrust } \\
\text { degree }\end{array}$ & $\begin{array}{c}\text { Trust } \\
\text { degree }\end{array}$ & $\begin{array}{c}\text { Distrust } \\
\text { degree }\end{array}$ \\
\hline Degree & 1324.2 & 78.8 & 1148.7 & 62.9 & 1041.5 & 56.6 & 966.0 & 57.8 & 909.9 & 52.7 & 792.2 & 47.1 & 737.5 & 46 \\
\hline PageRank & 731.7 & 50.6 & 826.9 & 48.3 & 802.4 & 44.9 & 741.6 & 40.9 & 687.7 & 43.7 & 645.9 & 46.8 & 601.7 & 44.3 \\
\hline HTMM & 930.7 & 47.4 & 894.2 & 43.6 & 825.4 & 39.6 & 779.5 & 38.3 & 740.4 & 36.2 & 690.9 & 38.8 & 646.3 & 36.4 \\
\hline
\end{tabular}

In order to further verify the HTMM method based on the hybrid trust network to identify the effectiveness of the user, the following analysis of the three affect the user's trust and distrust. As shown in Tab. 2, where Degree represents the degree-based algorithm, PageRank is based on the PageRank algorithm; HTMM represents the HTMMbased algorithm. Tab. 3 shows the average trust and distrust of Top 10, Top 20, Top 30, Top 40, Top 50, Top 80, and Top 100 in a hybrid trust network. The data in the Tab. 2 can be seen that the degree centrality algorithm to identify the user trust degree is the highest, but also have the highest degree of distrust; the trust of the HTMM algorithm is lower than that of the degree centrality algorithm, but its trust is less than that of the HTMM algorithm. However, the trust of HTMM algorithm is lower than that of degree-center algorithm; its trust is the lowest among the three kinds of user identification algorithms [16]. This is mainly because the degree centrality algorithm only considers the trust relationship under the hybrid trust network without considering the distrust relationship between users. The method mentioned in this paper also considers the relationship between trust and distrust, so that the impact of the users found in the high degree of trust at the same time, with the lowest degree of distrust. Therefore, the proposed HTMM based user identification method presented by this paper shows a high ability.

\section{CONCLUSION}

The influence of user identification based on trust is the key to enterprise's word-of-mouth marketing. However, trust and mistrust are independent and coexistent concepts, which together constitute the user trust network. Therefore, we need to consider both the user's trust and mistrust in two aspects, the study of hybrid trust network environment affect the user's identification method. In this paper, the structural properties of hybrid trust networks are studied by using the cumulative distribution of trust degree and distrust degree, the cumulative distribution of trust and distrust ratio and hybrid model. The study found that in a hybrid trust network, users with low trusts are less likely to gain the trust of other users, that is, most users are more likely to favor higher-confidence users rather than those who do not trust. In addition, hybrid trust networks exhibit heterogeneity based on the co-ordination results of hybrid trust networks. It is shown that nodes with small values are more likely to have nodes with high connectivity values, both of which show a negative correlation. Based on the PageRank algorithm, this paper combines the trust and distrust relationship, and puts forward the method of user recognition based on HTMM. Experiments were carried out on real data of Epinions.com, which included user trust and distrust. The experimental results show that the influence of user identification based on HTMM is that the user has a low degree of distrust while maintaining a high degree of trust. The research of this paper can provide decision- making basis for enterprises in the process of selection based on trusted users.

\section{Acknowledgment}

This work was supported by the National Natural Science Foundation of China (61472136, 61772196), the Hunan Provincial Focus Social Science Fund (2016ZDB006) and Hunan Provincial Social Science Achievement Review Committee results assessment identification project (Xiang Social Assessment 2016JD05). The authors gratefully acknowledge the financial support provided by the Key Laboratory of Hunan Province for New Retail Virtual Reality Technology (2017TP1026).

\section{REFERENCES}

[1] Stelzner, M. A. (2010). Social media marketing industry report. http://www.socialmediaexaminer.com/socialmediamarketing-industry-report-2010

[2] Kaplan, A. M. \& Haenlein, M. (2010). Users of the world, unite! The challenges and opportunities of Social Media. Business horizons, 53(1), 59-68. https://doi.org/10.1016/j.bushor.2009.09.003

[3] DuBois, T., Golbeck, J., \& Srinivasan, A. (2011). Predicting trust and distrust in social networks. Privacy, Security, Risk and Trust (PASSAT) and 2011 IEEE Third International Conference on Social Computing (SocialCom), 418-424. https://doi.org/10.1109/PASSAT/SocialCom.2011.56

[4] Kim, Y. A. \& Ahmad, M. A. (2013). Trust, distrust and lack of confidence of users in online social media-sharing communities. Knowledge-Based Systems, 37, 438-450. https://doi.org/10.1016/j.knosys.2012.09.002

[5] Lewicki, R. J., McAllister, D. J., \& Bies, R. J. (1998). Trust and distrust: New relationships and realities. Academy of management Review, 23(3), 438-458. https://doi.org/10.5465/amr.1998.926620

[6] Kim, Y. S. \& Tran, V. L. (2013). Assessing the ripple effects of online opinion leaders with trust and distrust metrics. Expert Systems with Applications, 40(9), 3500-3511. https://doi.org/10.1016/j.eswa.2012.12.058

[7] Leskovec, J., Huttenlocher, D., \& Kleinberg, J. (2010). Predicting positive and negative links in online social networks. Proceedings of the $19^{\text {th }}$ international conference on World Wide Web. ACM, 641-650. https://doi.org/10.1145/1772690.1772756

[8] Li, Y. M., Lai, C. Y., \& Chen, C. W. (2011). Discovering influencers for marketing in the blogosphere. Information Sciences, 181(23), 5143-5157. https://doi.org/10.1016/j.ins.2011.07.023

[9] Sherchan, W., Nepal, S., \& Paris, C. (2013). A survey of trust in social networks. ACM Computing Surveys (CSUR), 45(4), 1-47. https://doi.org/10.1145/2501654.2501661

[10] Berlmgerio, M., Coscia, M., Giannom, F., Monreale, A., \& Pedreschi, D. (2013). Multidimensional networks: foundations of structural analysis. World Wide Web, 16(5), 567-593. https://doi.org/10.1007/s11280-012-0190-4

[11] Liu, S., Jiang, C., Lin, Z., Ding, Y., Duan, R., \& Xu, Z. (2015). Identifying effective influencers based on trust for 
electronic word-of-mouth marketing: A domain-aware approach. Information Sciences, 306, 34-52. https://doi.org/10.1016/j.ins.2015.01.034

[12] Jiang, W., Xu, Y., Guo, H., \& Zhang, L. (2014). Multi agent system-based dynamic trust calculation model and credit management mechanism of online trading. Science China. Information science, 44(9), 1084-1101. https://doi.org/10.1360/N112013-00202

[13] Jiang, W. J., Zhong, L., Zhang, L. M., \& Shi, D. J. (2013). Dynamic cooperative multi-agent model of complex system based-on sequential action' logic. Chinese journal of computers, 36(5), 115-1124.

[14] Jiang, W. J., Zhang, L. M., \& Wang, P. (2009). Research on Grid Resource Scheduling Algorithm Based on MAS Cooperative Bidding Game. Chinese Science F, 52(8), 13021320. https://doi.org/10.1007/s11432-009-0151-4

[15] Jiang, W., Xu, Y., \& Zhang, L. (2013). Dynamic evolution model reuse complex knowledge-based component technology MAS. Systems Engineering Theory and Practice, 33(10), 2663-2673.

[16] Hamed, K., Bashah, I. N., \& Jamalul-lail, A. M. (2013). A unified trust model for pervasive environments-simulation and analysis. KSII Trans. on Internet \& Information Systems, 7(7), 1569-1584. https://doi.org/10.3837/tiis.2013.07.003

[17] Brocheler, M., Pugliese, A., \& Subrahmanian, V. S. (2011). Probabilistic subgraph matching on huge social networks. Proc. of the 2011 International Conference on Advances in Social Networks Analysis and mining, IEEE, Kaohsiung, Taiwan, 271-278. https://doi.org/10.1109/ASONAM.2011.78

[18] Zhu, Z. (2013). Discovering the influential users oriented to viral marketing based on online social networks. Physica A: Statistical Mechanics and Its Applications, 392(16), 34593469. https://doi.org/10.1016/j.physa.2013.03.035

[19] Probst, E., Grosswiele, D. K. L., Pfleger, D. K. R. (2013). Who will lead and who will follow: Identifying Influential Users in Online Social Networks. Business \& Information Systems Engineering, 5(3): 179-193. https://doi.org/10.1007/s12599-013-0263-7

[20] Aral, S. \& Walker, D. (2012). Identifying influential and susceptible members of social networks. Science, 337(6092), 337-341. https://doi.org/10.1126/science.1215842

[21] Gjoka, M., Kurant, M., Butts, C. T., \& Markopoulou, A. (2016). Walking in Facebook: A case study of unbiased sampling of OSNs. Proc. of the International Conference on Computer Communications (INFOCOM' 16), IEEE, San Diego, CA, USA, 1-9.

[22] Lu, D., Li, Q., Liao, S. S. (2015). A graph-based action network framework to identify prestigious members through member's prestige evolution. Decision Support Systems, 53, 44-54. https://doi.org/10.1016/j.dss.2011.12.003

[23] Chang, M. K., Cheung, W., \& Tang, M. (2013). Building trust online: Interactions among trust building mechanisms, Information \& Management, 50(7), 439-445. https://doi.org/10.1016/j.im.2013.06.003

\section{Contact information:}

\section{Weijin JIANG}

Institute of Big Data and Internet Innovation,

Mobile E-business Collaborative Innovation Center of Hunan Province,

Hunan University of Commerce, Changsha 410205, China

Key Laboratory of Hunan Province for New Retail Virtual Reality Technology,

Hunan University of Commerce, Changsha 410205, China

School of Computer Science and Technology,

Wuhan University of Technology, Wuhan 430073, China

jlwxjh@163.com

\section{Jiahui CHEN}

Corresponding author

Institute of Big Data and Internet Innovation,

Mobile E-business Collaborative Innovation Center of Hunan Province,

Hunan University of Commerce, Changsha 410205, China

810663304@qq.com

Yuhui XU

Institute of Big Data and Internet Innovation,

Mobile E-business Collaborative Innovation Center of Hunan Province, Hunan University of Commerce, Changsha 410205, China

363168449@qq.com 